\title{
GREEN'S THEOREM AND GREEN'S FUNCTIONS FOR CERTAIN SYSTEMS OF DIFFERENTIAL EQUATIONS*
}

\author{
BY
}

\section{MAX MASON}

The Green's function, defined originally for the potential equation, has been generalized to apply to the linear differential equation of second order of the elliptic type. $\dagger$ In the present paper the further generalization of Green's theorem and function to certain systems of differential equations will be made, and, the existence of the Green's functions being postulated, theorems regarding them will be established.

\section{$\S 1$. Green's Theorem.}

Consider the system of differential expressions

(1) $L_{i}\left(u_{1}, u_{2}, \cdots, u_{n}\right)=\Delta u_{i}+\sum_{j=1}^{n}\left\{a_{i j} \frac{\partial u_{j}}{\partial x}+b_{i j} \frac{\partial u_{j}}{\partial y}+c_{i j} u_{j}\right\} \quad(i=1,2, \cdots, n)$,

where $\Delta$ is the Laplace operator; $a_{i j}, b_{i j}, c_{i j}$ are continuous functions of $x, y$; and $a_{i j}, b_{i j}$ have continuous first derivatives.

Let $u_{i}, v_{i}$ be any functions of $x, y$ which, within a region $\Omega$ and on its boundary, possess at every point second derivatives with respect to $x$ and second derivatives with respect to $y$. The use of the ordinary formulae for integration by parts gives, after rearrangement of terms, the following Green's theorem for the system (1):

$$
\sum_{i=1}^{n} \iint_{(\Omega)}\left\{v_{i} L_{i}\left(u_{1}, \cdots, u_{n}\right)-u_{i} M_{i}\left(v_{1}, \cdots, v_{n}\right)\right\} d x d y=\int_{(S)}(Q d y-P d x),
$$

where $S$ is the boundary of the region $\Omega$, and

$$
M_{i}\left(v_{1}, \cdots, v_{n}\right)=\Delta v_{i}-\sum_{j=1}^{n}\left\{\frac{\partial\left(a_{j i} v_{j}\right)}{\partial x}+\frac{\partial\left(a_{j i} v_{j}\right)}{\partial y}-c_{j i} v_{j}\right\}
$$

* Presented to the Society September 1, 1903. Received for publication October 31, 1903.

†See Encyklopädie der mathematischen Wissenschaften, IIA7c (SommerFELD), \& 3. 


$$
\begin{aligned}
& P=\sum_{i=1}^{n}\left\{v_{i} \frac{\partial u_{i}}{\partial y}-u_{i} \frac{\partial v_{i}}{\partial y}+v_{i} \sum_{j=1}^{n} b_{i j} u_{j}\right\}, \\
& Q=\sum_{i=1}^{n}\left\{v_{i} \frac{\partial u_{i}}{\partial x}-u_{i} \frac{\partial v_{i}}{\partial x}+v_{i} \sum_{j=1}^{n} a_{i j} u_{j}\right\} .
\end{aligned}
$$

Applying the formulae :

$$
\begin{aligned}
& \frac{d y}{d s}=\frac{d x}{d n}=\cos (n, x), \\
& \frac{d x}{d s}=-\frac{d y}{d n}=-\cos (n, y),
\end{aligned}
$$

where $s$ is the length of arc along $S$ and $n$ the outward drawn normal, the following form is deduced:

(2) $\sum_{i=1}^{n} \iint_{(\Omega)}\left\{v_{i} L_{i}\left(u_{1}, \ldots, u_{n}\right)-u_{i} M_{i}\left(v_{1}, \ldots, v_{n}\right)\right\} d x d y$

$$
=\sum_{i=1}^{n} \int_{(S)}\left\{v_{i} \frac{\partial u_{i}}{\partial n}-u_{i} \frac{\partial v_{i}}{\partial n}+v_{i} \sum_{j=1}^{n}\left[a_{i j} \cos (n, x)+b_{i j} \cos (n, y)\right] u_{j}\right\} d s .
$$

The system of differential expressions $M_{i}\left(v_{1}, \ldots, v_{n}\right)$ will be called the $a d-$ joint system to $L_{i}\left(u_{1}, \cdots, u_{n}\right) .^{*}$ It follows that the relation is reciprocal, the system $L_{i}$ being adjoint to $M_{i}$. The conditions that the system $L_{i}$ be self adjoint are easily seen to be:

$$
\begin{gathered}
a_{i j}=-a_{j i}, \quad b_{i j}=-b_{j i}, \\
\frac{\partial a_{j i}}{\partial x}+\frac{\partial b_{j i}}{\partial y}=c_{j i}-c_{i j}
\end{gathered}
$$$$
(i, j=1,2, \cdots, n)
$$

§ 2. The Unique Determination of Solutions by their Boundary Values.

As an application of (2) let us consider under what conditions a set of solutions $\nmid$ of the system

$$
L_{i}\left(u_{1}, \cdots, u_{n}\right)=f_{i} \quad(i=1,2, \cdots, n)
$$

within a region $\Omega$, where the $f_{i}$ are given continuous functions of $x, y$, are uniquely determined by their boundary values. This is equivalent to asking under what conditions a set of solutions, not all zero, of the homogeneous system

* Professor Bôcher, to whom I am indebted for many valuable suggestions and criticisms, has called my attention to the introduction by JORDAN of adjoint systems of ordinary differential equations of the first order. JoRdaN, Cours d'analyse, vol. 3, p. 144.

+By a "set of solutions" within $\Omega$ we shall mean a set of functions which satisfy the equations at every point of $\Omega$, thus demanding the existence of the second derivatives and continuity of the first. 


$$
L_{i}\left(u_{1}, \cdots, u_{n}\right)=0
$$

which vanish on the boundary, can not exist.

Let the functions $u_{i}$ of (2) be a set of solutions of (4) vanishing on $S$, and choose $v_{i}=u_{i}$. Then from (2):

$-\sum_{i=1}^{n} \iint_{(\Omega)} u_{i} M_{i}\left(u_{1}, \cdots, u_{n}\right) d x d y=\sum_{i=1}^{n} \iint_{(\Omega)}\left\{\left(\frac{\partial u_{i}}{\partial x}\right)^{2}+\left(\frac{\partial u_{i}}{\partial y}\right)^{2}\right\} d x d y$

$$
+\sum_{i, j=1}^{n} \iint_{(\Omega)} u_{i}\left\{\frac{\partial\left(a_{j i} u_{j}\right)}{\partial x}+\frac{\partial\left(b_{j i} u_{j}\right)}{\partial y}-c_{j i} u_{j}\right\} d x d y=0 .
$$

But

$$
\begin{array}{r}
\sum_{i, j=1}^{n} \iint_{(\Omega)}\left\{u_{i} u_{j}\left(\frac{\partial a_{j i}}{\partial x}+\frac{\partial b_{j i}}{\partial y}-c_{j i}\right)+u_{i}\left(a_{j i} \frac{\partial u_{j}}{\partial x}+b_{j i} \frac{\partial u_{j}}{\partial y}\right)\right\} d x d y \\
=\frac{1}{2} \sum_{i, j=1}^{n} \iint_{(\Omega)} u_{i} u_{j}\left(\frac{\partial a_{j b}}{\partial x}+\frac{\partial b_{j i}}{\partial y}-2 c_{j i}\right) d x d y
\end{array}
$$

for, since the functions $u_{i}$ vanish on the boundary,

$$
\iint_{(\Omega)} a_{j i} u_{i} \frac{\partial u_{j}}{\partial x} d x d y=-\iint_{(\Omega)}\left(u_{i} u_{j} \frac{\partial a_{j i}}{\partial x}+u_{j} a_{j i} \frac{\partial u_{i}}{\partial x}\right) d x d y
$$

and similarly for the other terms. The above equation then becomes:

$$
\begin{aligned}
\sum_{i=1}^{n} \iint_{(\Omega)}\left\{\left(\frac{\partial u_{i}}{\partial x}\right)^{2}+\left(\frac{\partial u_{i}}{\partial y}\right)^{2}\right\} d x d y & \\
& +\frac{1}{2} \sum_{i, j=1}^{n} \iint_{(\Omega)} u_{i} u_{j}\left(\frac{\partial a_{j i}}{\partial x}+\frac{\partial b_{j i}}{\partial y}-2 c_{j i}\right) d x d y=0 .
\end{aligned}
$$

The solutions of the homogeneous system (4) which vanish on the boundary are therefore all zero, if within $\Omega$

$$
\sum_{i, j=1}^{n}\left\{u_{i} u_{j}\left(\frac{\partial a_{j i}}{\partial x}+\frac{\partial b_{j i}}{\partial y}-2 c_{j i}\right)\right\}>0 .
$$

We have therefore the theorem:

The solutions of the system

$$
L_{i}\left(u_{1}, \cdots, u_{n}\right)=f_{i} \quad(i=1,2, \cdots, n)
$$

within a region $\Omega$ are uniquely determined by their values on the boundary of $\Omega$ provided that the coefficients $a_{j i}, b_{j i}, c_{j i}$ are such that 


$$
\sum_{i, j=1}^{n} i, j\left\{u_{i} u_{j}\left(\frac{\partial a_{j i}}{\partial x}+\frac{\partial b_{j i}}{\partial y}-2 c_{j i}\right)\right\}
$$

is a positive definite form.

§ 3. Green's Functions and the Law of Reciprocity.

By Green's functions of the system of differential equations

$$
L_{i}\left(u_{1}, \cdots, u_{n}\right)=0
$$$$
(i=1,2, \cdots, n)
$$

for a region $\Omega$ of the $x, y$ plane, we shall understand $n$ sets of $n$ functions of $x, y, \xi, \eta, G_{k i}(x, y, \xi, \eta)$, which have the following properties:

Each set $G_{k i}(i=1,2, \ldots, n)$ is, in the variables $x, y, a$ solution of (4) within the region formed by excluding from $\Omega$ the point $x=\xi, y=\eta$.

The functions have thc form

$$
\begin{gathered}
G_{k i}=R_{k i}(x, y, \xi, \eta) \\
G_{i i}=\log \sqrt{(x-\xi)^{2}+(y-\eta)^{2}}+R_{i i}(x, y, \xi, \eta),
\end{gathered}
$$

where the functions $R$ possess second derivatives with respect to $x$ and $y$ for all points within $\Omega$ and on the boundary.

Each function vanishes identically in $\xi, \eta$ for values of $x, y$ on the boundary.

If the coefficients of the system (4) satisfy the condition of the theorem stated at the close of the preceding paragraph, the Green's functions are uniquely determined. For if two sets $G_{k i}^{(1)}, G_{k i}^{(2)}$ existed, the functions

$$
u_{i}=G_{k i}^{(1)}-G_{k i}^{(2)}
$$

for any $k$, would be solutions of (4) within the entire region $\Omega$ which vanish on the boundary.

Denote a set of Green's functions of the adjoint system

$$
M_{i}\left(v_{1}, \cdots, v_{n}\right)=0 \quad(i=1,2, \cdots, n)
$$

by $H_{k i}(x, y, \xi, \eta)$.

The Green's functions $G_{k i}$ of the system (4) and $H_{k i}$ of the adjoint system (5) nbey the following law of reciprocity:

$$
G_{k i}(x, y, \xi, \eta)=H_{i k}(\xi, \eta, x, y) .
$$

To prove this relation consider any two points $\xi, \eta$ and $\xi^{\prime}, \eta^{\prime}$ within $\Omega$, and substitute in (2) for $u_{1}, u_{2}, \ldots, u_{n}$ the functions $G_{k i}(x, y, \xi, \eta)(i=1,2, \ldots, n)$ and for $v_{1}, v_{2}, \cdots, v_{n}$ the functions $H_{i k}\left(x, y, \xi^{\prime}, \eta^{\prime}\right)(k=1,2, \cdots, n)$. Apply (2) to the region $\Omega^{\prime}$ bounded by the boundary of $\Omega$ and two circles $c, c^{\prime}$ within $\Omega$, of radii $r, r^{\prime}$ about $\xi, \eta$ and $\xi^{\prime}, \eta^{\prime}$. Since the functions $G$ form a solution 
of (4) and the functions $H$ a solution of (5) within $\Omega^{\prime}$, and all the functions vanish on the boundary, (2) becomes:

$$
\begin{aligned}
& \sum_{j=1}^{n} \int_{\left(c+c^{\prime}\right)}\left\{H_{i j}\left(x, y, \xi^{\prime}, \eta^{\prime}\right) \frac{\partial G_{k i}(x, y, \xi, \eta)}{\partial n}\right. \\
& \left.-G_{k i}(x, y, \xi, \eta) \frac{\partial H_{i j}\left(x, y, \xi^{\prime}, \eta^{\prime}\right)}{\partial n}\right\} d s \\
& +\sum_{j, h=1}^{n} \int_{\left(c+c^{\prime}\right)} G_{k h}(x, y, \xi, \eta) H_{i j}\left(x, y, \xi^{\prime}, \eta^{\prime}\right)\left\{a_{j h} \cos (n, x)\right. \\
& \left.+b_{j h} \cos (n, y)\right\} d s=0 .
\end{aligned}
$$

This equation holds for all $r, r^{\prime}$ however small. Replacing $n, d s$ by $-r, r d \theta$ on $c$ and by $-r^{\prime}, r^{\prime} d \theta^{\prime}$ on $c^{\prime}$ we have:

where

$$
\begin{aligned}
\int_{0}^{2 \pi} H_{i k}\left(x, y, \xi^{\prime}, \eta^{\prime}\right) \frac{\partial G_{k l}(x, y, \xi, \eta)}{\partial r} r d \theta & \\
& -\int_{0}^{2 \pi} G_{k i}(x, y, \xi, \eta) \frac{\partial H_{i i}\left(x, y, \xi^{\prime}, \eta^{\prime}\right)}{\partial r^{\prime}} r^{\prime} d \theta^{\prime}+R+R^{\prime}=0
\end{aligned}
$$

$$
\lim _{r=0} R=0, \quad \lim _{r^{\prime}=0} R^{\prime}=0,
$$

for the products of $r$ or $r^{\prime}$ with all terms except $\partial G_{k k} / \partial r$ and $\partial H_{i i} / \partial r^{\prime}$ approach zero as $r$ or $r^{\prime}$ approaches zero, since these are the only terms that contain more than a logarithmic singularity. Passing to the limit $r=0, r^{\prime}=0$ we obtain by well known methods*

$$
H_{i k_{i}}\left(\xi, \eta, \xi^{\prime}, \eta^{\prime}\right)=G_{k i}\left(\xi^{\prime}, \eta^{\prime}, \xi, \eta\right),
$$

which is the law of reciprocity.

From this law the theorem is evident:

If a set of Green's functions exists for the system (4) and for the adjoint system (5), then these sets are uniquely determined.

If the system (4) is self adjoint the law of reciprocity becomes

$$
G_{k i}(x, y, \xi, \eta)=G_{i k}(\xi, \eta, x, y),
$$

and it follows that the set is uniquely determined.

\section{$\S 3$. Integral Representation of Solutions.}

Let the $u_{i}$ in (2) be a set of solutions of the equations

$$
L_{i}\left(u_{1}, \ldots, u_{n}\right)=f_{i}
$$$$
(i=1, i, \cdots, n) \text {, }
$$

* See e. g. Picard, Trailé d'analyse, vol. 2, p. 15. 
within $\Omega$, and denote the values of $u_{i}$ on the boundary $S$ of $\Omega$ by $\bar{u}_{i}$. For $v_{i}$ in (2) take the Green's functions $H_{k i}(x, y, \xi, \eta)$ of the adjoint system (5). Applying (2) to the region $\Omega^{\prime}$ bounded by $S$ and a circle $c$, within $\Omega$, of radius $r$ about $\xi, \eta$ we have, after replacing $n, d s$ on $c$ by $-r, r d \theta$,

$\sum_{i=1}^{n} \iint_{\left(\Omega^{\prime}\right)} H_{k i} f_{i} d x d y=-\sum_{i=1}^{n} \int_{(S)} \bar{u}_{i} \frac{\partial H_{k i}}{\partial n} d s$

where

$$
-\sum_{i=1}^{n} \int_{0}^{2 \pi}\left\{H_{k i} \frac{\partial u_{i}}{\partial r}-u_{i} \frac{\partial H_{k i}}{\partial r}+R\right\} r d \theta
$$

$$
\lim _{r=0}(R r)=0 \text {. }
$$

In the limit $r=0$ the term containing $\partial H_{k k} / \partial r$ is the only one which gives a value different from zero to the last integral. In the same manner as for a single equation we have then in the limit:

$$
2 \pi u_{k}(\xi, \eta)=\sum_{i=1}^{n} \int_{(S)} \bar{u}_{i} \frac{\partial H_{k i}}{\partial n} d s+\sum_{i=1}^{n} \iint_{(\Omega)} H_{k i} f_{i} d x d y .
$$

The integral formula (6) gives the set of solutions of (3) within $\Omega$ which assume the values $\bar{u}_{i}$ on the boundary.

MassachusetTs Institute of TechNology. 